\title{
Ferromagnetic Resonance in CdTe:Cr Single Crystals Containing Dopant-Related Macrodefects
}

\author{
V.D. Popovych ${ }^{a}$, M. Kuzma ${ }^{b, *}$, N. $\mathrm{SkOP}^{b}$, I. Stefaniuk ${ }^{c}$ And B. CienieK ${ }^{c}$ \\ ${ }^{a}$ Department of Machine Science and Fundamental Technologies, Drogobych State Pedagogical University, \\ 24 Ivan Franko str., 82100 Drogobych, Ukraine \\ ${ }^{b}$ Faculty of Mathematics and Natural Sciences, Department of Biophysics, University of Rzeszów, \\ S. Pigonia 1, 35-959 Rzeszów, Poland \\ ${ }^{c}$ Faculty of Mathematics and Natural Sciences, Department of Experimental Physics, University of Rzeszów, \\ S. Pigonia 1, 35-959 Rzeszów, Poland
}

We study CdTe:Cr single crystals grown by the physical vapor transport method from pre-synthesized $(\mathrm{Cd}, \mathrm{Cr}) \mathrm{Te}$ alloys with 5 at.\% of chromium nominal content. Macrodefects in the form of (111)-oriented thin platelets of dopant-related second phases were detected by scanning electron microscopy patterning of the chemically treated surfaces of the crystals. Magnetic properties of the crystals were investigated by ferromagnetic resonance using X-band Bruker spectrometer $(9.43 \mathrm{GHz})$. Their ferromagnetic resonance spectra show several broad lines, which position depends on the orientation of the sample in relation to the external magnetic field in spectrometer. The angular dependences of ferromagnetic resonance spectra are interpreted in the frame of shape anisotropy of ferromagnetic resonance of the planar defects embedded in a weak magnetic CdTe matrix.

DOI: 10.12693/APhysPolA.132.34

PACS/topics: $61.72 .-\mathrm{y}, 68.37 . \mathrm{Hk}, 76.50 .+\mathrm{g}$

\section{Introduction}

One of the most challenging tasks of material science in the field of spintronics is to obtain diluted ferromagnetic semiconductors (DMS) exhibiting ferromagnetic properties up to above the room temperature [1]. Among other compounds, considerable efforts were put to induce hightemperature ferromagnetic properties into II-VI chalcogenides by partial replacement of their cations with appropriate number of magnetic transition-metal (TM) ions. Substitutional doping with chromium was theoretically predicted to be the most preferable due to the fact that the mechanism of both carrier mediated and ionion interactions are ferromagnetic in Cr doped II-VI [24]. Starting from the first experimental confirmation of the above prediction by Saito et al. [5], main efforts were focused on heavily chromium doped $\mathrm{Cr}$-doped $\mathrm{ZnTe}$ epitaxial layers (e.g. [6-8]), whereas data about CdTebased material much less occurred. Although preparation of $\mathrm{Cd}_{1-x} \mathrm{Cr}_{x}$ Te single crystal with $x \leq 0.05$ was reported and their room-temperature ferromagnetic properties were proved by magnetization studies [9, 10], the nature of ferromagnetism in CdTe:Cr still appeared controversial. As in the case of other DMS doped with transition metals [11], there exist two different approaches concerning this problem. According to the first one, the appearance of room-temperature ferromagnetism was explained by ferromagnetic interaction of double ionized $\mathrm{Cr}$ atoms, randomly substituting metal ones in II-VI lattice [5-7]. Contrary, the other suggests that ferromag-

*corresponding author; e-mail: kuzma@univ.rzeszow.pl netic ordering originates from Cr-related second phases buried in the host matrix [12-14]. Our previous structural investigation confirmed the last supposition. Doping induced crystallographic phase separation was observed, resulting in the presence of CrTe-based defects in the form of sets of needle-like aggregates aligned parallel to the $\{111\}$ crystallographic planes of zinc-blende structure [15]. It has been determined by energy-dispersive X-ray (EDX) profiling and high-angle annular dark-field (HAADF) elemental mapping that their chemical composition corresponds well with that of $\mathrm{Cr}_{3} \mathrm{Te}_{4}$ phase [16], which is high-temperature ferromagnetic with the Curie temperature ranging from 315 to $340 \mathrm{~K}$ according to the literature data [17].

In the present paper, the results of electron magnetic resonance (EMR) measurements of heavily doped CdTe:Cr single crystals are presented. This technique is known to be a powerful tool to study the magnetic properties of great variety of materials, including Cr-doped IIIV compounds. However, the only investigations of the crystals with relatively low dopant concentration have been performed so far, aiming mainly to determine the contribution of Cr-related point defects into the resulting electron paramagnetic resonance (EPR) signal of the investigated samples and to identify their charge states and symmetry in the II-IV lattice [18-20]. As to our knowledge, the effect of doping induced phase separation in TM-doped II-IV crystals on their ferromagnetic resonance (FMR) spectra will be reported for the first time.

\section{Experimental}

CdTe:Cr crystals were grown by the modified physical vapor transport (PVT) method using pre-synthesized polycrystalline ingots as a charge. Details and condi- 
tions of the growth method and charge synthesis process were described elsewhere [15]. The results, presented in this paper, refer to the crystals grown from the charge with 5 at. $\%$ of chromium nominal content. As-grown crystal boule were cleaved into the discs along $\{110\}$ crystallographic planes. For the EMR measurements a specimen was cut from the disc by a wire saw in the form of rectangular parallelepiped in such a way that two side surfaces were (110)-oriented, and another two were (111)-oriented (Fig. 1). The last ones were prepared by lapping and polishing with diamond powder followed by chemo-mechanical polishing with bromine solution in the ethyleneglycol-dimethylformamide mixture in order to remove the damaged layer, while the (110)-oriented side surfaces were directly chemo-mechanically polished. Morphology and composition of the surfaces were studied by means of Tescan Vega 3 scanning electron microscope (SEM) equipped with Bruker Quantax EDX microanalyser. EMR spectra were recorded using Bruker Elexys E580 spectrometer operating at $9.4 \mathrm{GHz}$ (X-band). Measurements were carried out in the $300-360 \mathrm{~K}$ temperature range controlled by nitrogen cryostat system.
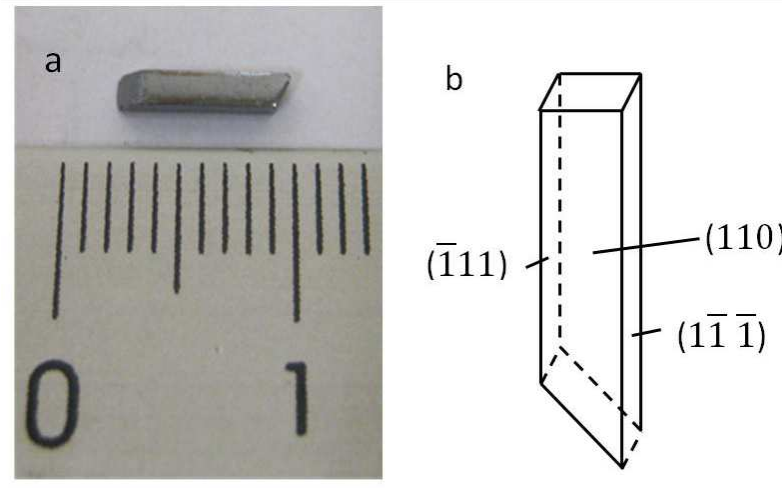

Fig. 1. (a) Picture of the sample for FMR measurements, prepared from the CdTe:Cr single crystal with 5 at.\% of chromium nominal content, (b) schematic view of the sample with indication of the orientations of its side surfaces.

\section{Results and discussion}

On the (110)-oriented surfaces of the investigated samples we expectedly observed the same "unusual" defects in the form of parallel $\mathrm{Cr}_{3} \mathrm{Te}_{4}$ needles, which were reported earlier $[15,16]$. At the same time, on the (111)oriented samples facets features in the form of polygons were seen in the course of SEM patterning in addition to the needles (Fig. 2). Therefore, it can be suggested that the defects are actually thin platelets, which appear as the needles when normally intersect with (110)-type crystalline planes.

Angular dependences of magnetic resonance at $300 \mathrm{~K}$ are presented in Fig. 3 for the three selected orientations of the samples in relation to the external magnetic field $\boldsymbol{B}_{0}$, defined by an angle $\alpha$ between the field direction

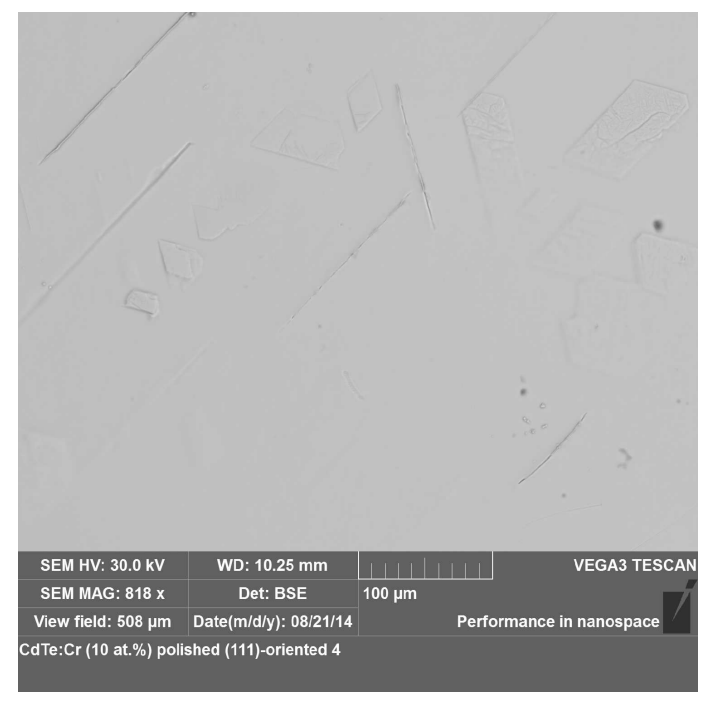

Fig. 2. SEM images of $\mathrm{Cr}_{3} \mathrm{Te}_{4}$ planar defects on the (111)-oriented surface of the CdTe:Cr single crystal with 5 at.\% of chromium nominal content.

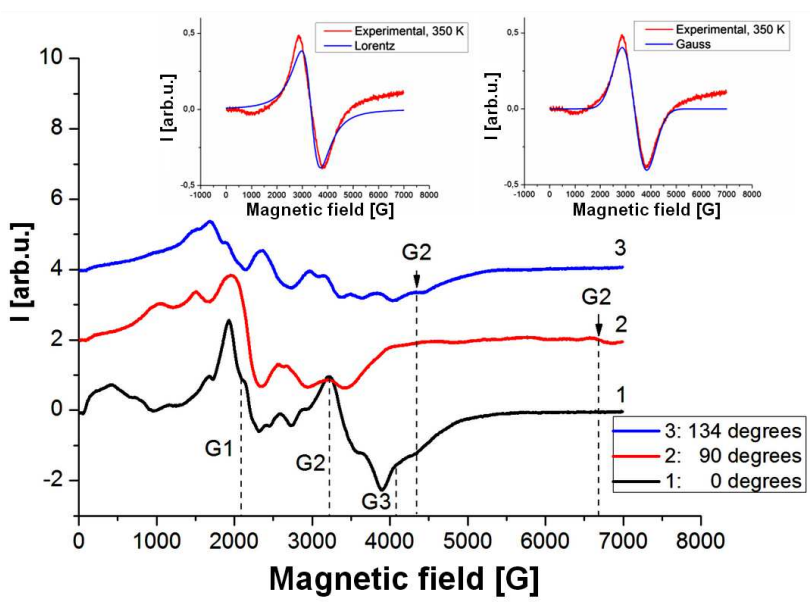

Fig. 3. Angular dependences of FMR spectrum of the CdTe:Cr single crystal with 5 at. $\%$ of chromium nominal content at $300 \mathrm{~K}$. The insets show Gaussian and Lorentzian fitting of the EPR spectrum of this crystal at $350 \mathrm{~K}$.

and (111)-oriented facet of the sample (see Fig. 1b). It is seen that the spectra consist of several lines. The results of deconvolution of the room-temperature spectrum for $\alpha=0^{\circ}$ into Gaussian lines indicate the presence of five individual components. At the same time, at higher temperatures the spectra are much simple for any sample orientation, and at $350 \mathrm{~K}$ the resonance spectrum is well approximated by three Gaussian lines. These lines at room temperature are labelled in Fig. 3 as G1, G2, G3 and constitute bases lines of the spectra. At room and at lower temperatures the spectra can be described by the FMR formalism [21].

In order to understand the complex structure of the obtained room-temperature spectra as well as their angular 
dependences, the contribution of ferromagnetic $\mathrm{Cr}_{3} \mathrm{Te}_{4}$ phases should be taken into account. It is obvious that since second phase particles appear in the form of thin platelets embedded into the CdTe:Cr matrix parallel to its $\{111\}$ crystallographic planes, their shape anisotropy results in different resonance field for different $\alpha$ values. In our case, due to the demagnetization field $N=\left(N_{x}, N_{y}, N_{z}\right)$, the value of resonance field strongly depends on the orientation of the external magnetic field in relation to the plane of the platelets [22]:

$$
\begin{aligned}
& \left(\frac{\omega}{\gamma}\right)^{2}=\left(H \cos \left(\theta-\theta_{H}\right)+H_{\text {eff }} \cos (2 \theta)\right) \\
& \quad \times\left(H \cos \left(\theta-\theta_{H}\right)+H_{\text {eff }} \cos ^{2} \theta\right)+\left(\frac{1}{\gamma T_{2}}\right)^{2},
\end{aligned}
$$

where $\gamma=\frac{e}{2 m_{e}} ; \theta$ and $\theta_{H}$ are the angles, defined in Fig. $4 \mathrm{a} ; \omega_{0}=2 \pi \nu$ is the circular frequency of the FMR spectrum. This formula is simplified for the two limiting cases [23]:

1. $\boldsymbol{B}_{0}$ is parallel to the platelets, the $x z$ planes, then $N_{x}=N_{z}=0, N_{y}=4 \pi$, and

$$
\omega_{0}=\gamma \sqrt{B_{0}\left(B_{0}+\mu_{0} M\right)} \text {. }
$$

2. $\boldsymbol{B}_{0}$ is perpendicular to the platelets, the $x z$ planes, then $N_{x}=N_{y}=0, N_{z}=4 \pi$, and

$$
\omega_{0}=\gamma\left(B_{0}-\mu_{0} M\right) \text {. }
$$

In Eqs. (2) and (3) $M$ is the magnetization field. Both of these cases are illustrated in Fig. $4 \mathrm{~b}$ and d, respectively.

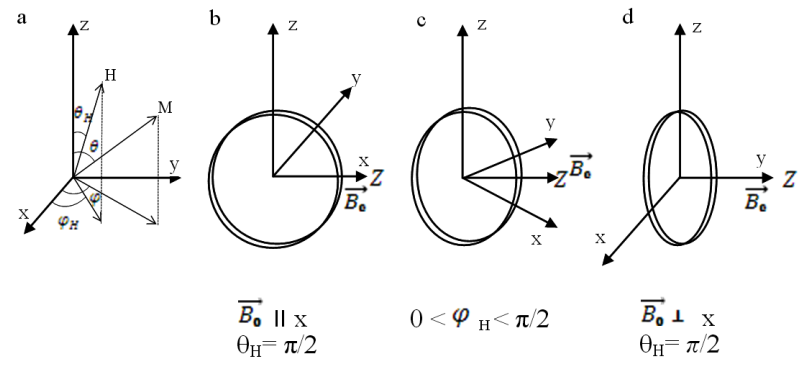

Fig. 4. (a) Presentation of magnetisation and magnetic field vectors in spherical coordinate system for ferromagnetic resonance, (b) and (d) "in-plane" and "out-of plane" configurations for FMR measurements, respectively, (c) intermediate configurations. The $x y z$ is the reference system connected with the sample.

The planar defects under consideration are aligned along the $\{111\}$ crystallographic planes of zinc-blende structure. There are eight different $\{111\}$ planes in elementary cell of CdTe, which are forming two tetrahedral intersecting each other (Fig. 5). Equations (2) and (3) were obtained by Kittel [23] for the particles having the shape of circular disks. Assuming a rectangular shape of the planar defects (which is reasonable assumption for the studied second phase particles), four different arrangements of the defects in relation to the applied magnetic field $\boldsymbol{B}_{0}$ and to the high frequency field $\boldsymbol{b}_{1}$ in the EPR spectrometer can be distinguished (Fig. 6). According to [24], in such a case in parallel configuration

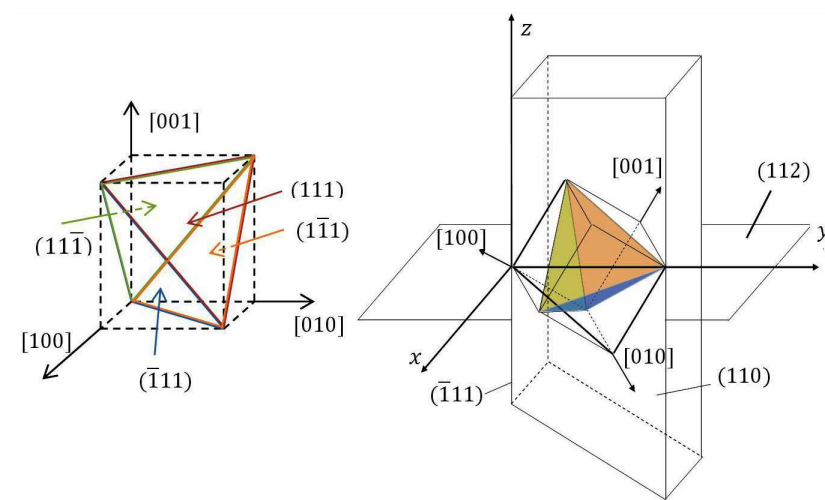

Fig. 5. Elementary cell of CdTe (a) and its crystallographic position in relation to the facets of the sample for FMR measurements (b). Second tetrahedron is not shown in order to avoid overloading of the figure.
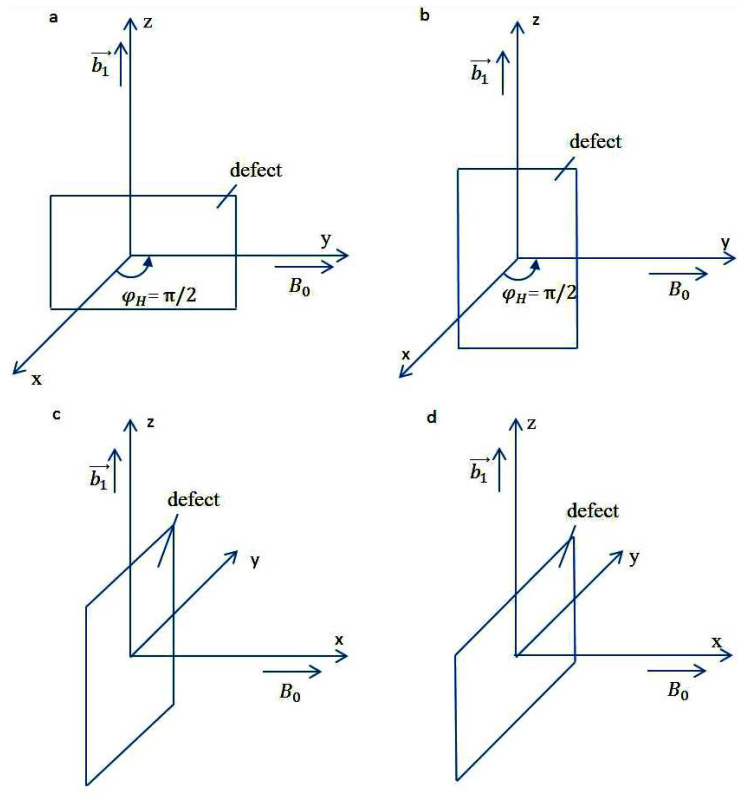

Fig. 6. Four possible orientations of the rectangular plane defects in relation to the magnetic field $\boldsymbol{B}_{0}$ and the microwave magnetic field $\boldsymbol{b}_{1}$.

$\left(\alpha=0^{\circ}\right)$ two resonance lines are located in the range of small magnetic fields, depending on the horizontal or vertical orientation of the defects (Fig. 6a,b), whereas for perpendicular geometry $\left(\alpha=90^{\circ}\right)$, presented in Fig. 6c and $\mathrm{d}$, two lines are situated in almost the same position at higher field for both orientations of the defects.

In our FMR experiments at $\alpha=0^{\circ}$ two planes, (111) and $(1 \overline{1} \overline{1})$, are parallel to the magnetic field $\boldsymbol{B}_{0}$, which is applied along $x$ axis in Fig. 4. The magnetic field is rotated in (112) plane during measurements of the angular dependences. The angles formed by the remaining crystallographic planes of $\{111\}$-type with the magnetic field direction are listed in Table I. Therefore, the FMR spectrum should contain lines with the resonance field corresponding to the specific $\varphi_{H}$ angles. 
TABLE I

The angles between the external magnetic field $\boldsymbol{B}_{0}$ and the plane of $\mathrm{Cr}_{3} \mathrm{Te}_{4}$ macrodefects.

\begin{tabular}{c|c|c|c|c}
\hline \hline \multirow{2}{*}{$\begin{array}{c}\text { Sample } \\
\text { orientation }\end{array}$} & \multicolumn{4}{|c}{ Parallel crystallographic planes } \\
\cline { 2 - 5 }$\alpha$ & $(\overline{1} 11)$ & $(111)$ & $(1 \overline{1} 1)$ & $(11 \overline{1})$ \\
& $(1 \overline{1} \overline{1})$ & $(\overline{1} \overline{1} \overline{1})$ & $(\overline{1} 1 \overline{1})$ & $(\overline{1} \overline{1} 1)$ \\
\hline $0^{\circ}$ & $0^{\circ}$ & $54^{\circ} 7^{\prime}$ & $0^{\circ}$ & $54^{\circ} 7^{\prime}$ \\
$90^{\circ}$ & $90^{\circ}$ & $19^{\circ} 28^{\prime}$ & $19^{\circ} 28^{\prime}$ & $19^{\circ} 28^{\prime}$
\end{tabular}

For the position of the sample at $\alpha=90^{\circ}$ the angles between $\{111\}$-oriented defects and magnetic field are changed (Table I). One can see from Table I that the crys-

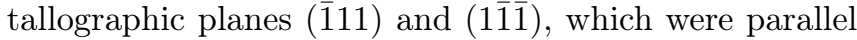
to the magnetic field at $\alpha=0^{\circ}$, became perpendicular to its direction at $\alpha=90^{\circ}$. Therefore, the resonance line of the defects, lying in these planes, should have extremely low value of the resonance field. This field will increase continuously with rotating the sample, and at $\alpha=90^{\circ}$ the resonance field achieves extremely high value. In the FMR spectra, presented in Fig. 3, the line denoted as G2 fulfils the above conditions. Considering Eqs. (2) and (3) for this line (taking values $H=3230 \mathrm{G}$ and $H=6700 \mathrm{G}$ for parallel and perpendicular orientation of defect, respectively) the magnetization $4 \pi M$ is equal to $1.42 \mathrm{~T}$. Such great value possibly follows from very good single crystallinity of CrTe defects studied. Further increase of the angle between the magnetic field and the plane of these defects to $\alpha=134^{\circ}$ decreases resonance field for the line G2, as it is shown in Fig. 3, spectrum 3. Similarly, the defects, oriented along the crystallographic

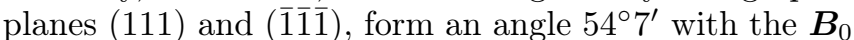
at $\alpha=0^{\circ}$ and should have greater resonance field value comparing with that of $\mathrm{G} 2$ at $\alpha=0^{\circ}$. It is reasonable to suppose that they create peak denoted as G3 in Fig. 3, spectrum 1.

\section{Conclusions}

Planar defects in the form of thin $\mathrm{Cr}_{3} \mathrm{Te}_{4}$ platelets aligned parallel to the $\{111\}$ crystallographic planes of zinc-blende structure were detected by electron microscopy in highly doped CdTe:Cr single crystals, grown by the modified PVT method. FMR measurements of the sample from the investigated crystal revealed complex character of their room-temperature spectra with a number of broad lines in the wide range of resonance field, which is not typical for CdTe single crystals. Angular dependences of the obtained FMR spectra were qualitatively explained considering shape anisotropy of the observed ferromagnetic defects, and corresponding resonance peaks, originating from these defects, were tentatively identified.

\section{References}

[1] T. Dietl, H. Ohno, Rev. Mod. Phys. 86, 187 (2014).

[2] J. Blinowski, P. Kacman, J.A. Majewski, J. Cryst. Growth 159, 972 (1996).
[3] H. Shoren, F. Ikemoto, K. Yoshida, N. Tanaka, K. Motizuki, Physica E 10, 242 (2001).

[4] N.A. Noor, S. Ali, A. Shaukat, J. Phys. Chem. Solids 72, 836 (2011).

[5] H. Saito, V. Zayets, S. Yamagata, K. Ando, Phys. Rev. Lett. 90, 207202 (2003).

[6] S. Kuroda, N. Ozaki, N. Nishizawa, T. Kumekawa, S. Marcet, K. Takita, Sci. Technol. Adv. Mater. 6, 558 (2005).

[7] W.G. Wang, K.J. Yee, D.H. Kim, K.J. Han, X.R. Wang, C. Ni, T. Moriyama, A. Mathew, R. Opila, T. Zhu, J.Q. Xiao, Phys. Rev. B 77, 155207 (2008).

[8] D. Soundararajan, D. Mangalaraj, D. Nataraj, L. Dorosinskii, J. Santoyo-Salazar, J.-M. Ko, Curr. Appl. Phys. 10, 771 (2010).

[9] K.Y. Ko, M.G. Blamire, Appl. Phys. Lett. 88, 172101 (2006).

[10] M.R. Begam, N.M. Rao, S. Kaleemulla, N.S. Krishna, M. Kuppan, G. Krishnaiah, J. Subrahmanyam, Mater. Sci. Semicond. Proc. 18, 146 (2014).

[11] T. Dietl, K. Sato, T. Fukushima, A. Bonanni, M. Jamet, A. Barski, S. Kuroda, M. Tanaka, P.N. Hai, H. Katayama-Yoshida, Rev. Mod. Phys. 87, 1311 (2015).

[12] S. Kuroda, N. Nishizawa, K. Takita, M. Mitome, Y. Bando, K. Osuch, T. Dietl, Nat. Mater. 6, 440 (2007).

[13] M.G. Sreenivasan, K.L. Teo, X.Z. Cheng, M.B.A. Jalil, T. Liew, T.C. Chong, A.Y. Du, T.K. Chan, T. Osipowicz, J. Appl. Phys. 102, 053702 (2007).

[14] H. Kobayashi, Y. Nishio, K. Kanazawa, S. Kuroda, M. Mitome, Y. Bando, Physica B 407, 2947 (2012).

[15] V.D. Popovych, P. Sagan, M. Bester, B. Cieniek, M. Kuzma, J. Cryst. Growth 426, 173 (2015).

[16] V.D. Popovych, P. Sagan, Acta Phys. Pol. A 129, 49 (2016).

[17] J. Dijkstra, H.H. Weitering, C.F. van Bruggen, C. Haas, R.A. de Groot, J. Phys. Condens. Matter 1, 9141 (1989).

[18] J.T. Vallin, G.D. Watkins, Phys. Rev. B 9, 2051 (1974).

[19] M.Z. Cieplak, M. Godlewski, J.M. Baranowski, Phys. Status Solidi B 70, 323 (1975).

[20] M.E.J. Boonman, W. Mac, A. Twardowski, A. Wittlin, P.J.M. van Bentum, J.C. Maan, M. Dziemianiuk, Phys. Rev. B 61, 5358 (2000).

[21] Ferromagnetic Resonance, Ed. S.V. Vonsovskii, Pergamon Press, Oxford 1966.

[22] Ferromagnetic Resonance - Theory and Application, Ed. O. Yalcin, InTech, Rijeka 2013.

[23] C. Kittel, Introduction to Solid State Physics, Wiley, New York 2005.

[24] U. Netzelmann, J. Appl. Phys. 68, 1800 (1990). 